\title{
Diferenças regionais no acesso a cirurgia cardiovascular no Brasil, 2002 -2010
}

\author{
Regional differences in access to coronary bypass surgery \\ in Brazil, 2002-2010
}

Francisco Viacava ${ }^{1}$

Silvia Porto ${ }^{2}$

Josué Laguardia ${ }^{1}$

Rodrigo da Silva Moreira ${ }^{1}$

Maria Alícia Dominguez Ugá ${ }^{2}$

${ }^{1}$ Laboratório de

Informações em Saúde,

Centro de Informação

Científica e Tecnológica,

Fiocruz. Av Brasil 4365,

Manguinhos. 21045-900

Rio de Janeiro RJ.

fviacava@gmail.com

${ }^{2}$ Depto Administração e

Planejamento em Saúde,

Escola Nacional de Saúde

Pública, Fiocruz.

\begin{abstract}
The hospitalization rates for angioplasty and coronary bypass surgery have been used as proxies for access to highly specialized services. The scope of this study is to analyze the evolution of these rates and discuss what are the possible causes associated with regional inequalities. Standardized rates of angioplasty and coronary bypass surgery by age and sex per 100,000 inhabitants aged 20 and over, in the period from 2002 to 2010 were calculated. Comparison with international data shows that Brazil has lower rates than those observed in OECD countries. In Brazil, the standardized rates of hospitalization for angioplasty in the population aged 20 and over showed an upward trend, rising from 27.5 per 100,000 in 2002 to 39 in 2010. When mortality rates by age and sex from different geographical regions were compared, besides the marked differences in the north - south axis, what is notable is the maintenance of a stable pattern of these rates and regional differences over the period analyzed. The definition of regional health care networks for cardiac surgery is an important strategy to ensure the quality of care, optimization of operating costs and reduction of inequalities in access to healthcare between Brazilian regions.
\end{abstract}

Key words Assessment of Health Services, Access, Coronary bypass surgery, Angioplasty
Resumo As taxas internação por angioplastia e cirurgia de revascularização vêm sendo usadas como proxies de acesso a serviços de alta complexidade. O objetivo é analisar sua evolução e discutir quais seriam as possíveis causas associadas às desigualdades regionais. Foram calculadas as taxas padronizadas de realização de angioplastia e cirurgia de revascularização por sexo e idade por 100 mil habitantes de 20 anos e mais, no período 2002 a 2010. A comparação com os dados internacionais mostra que o Brasil tem taxas menores que as observadas nos países da OECD. No Brasil, as taxas padronizadas de internação por angioplastia na população de 20 anos ou mais apresentaram uma tendência de crescimento, passando de 27,5 por 100 mil habitantes em 2002 para 39 por 100 mil em 2010. Na comparação das taxas padronizadas por idade e sexo entre as grandes regiões do Brasil, além das diferenças marcantes no eixo Norte - Sul, o que chama atenção é que mantenham um padrão estável e também as diferenças regionais. A constituição de redes assistenciais regionais hierarquizadas para cirurgias cardíacas constitui uma estratégia importante para: garantir a qualidade do cuidado, a optimização dos custos operacionais e reduzir as desigualdades no acesso entre as regiões brasileiras.

Palavras-chave Avaliação de serviços de saúde, Acesso, Revascularização do miocárdio, Angioplastia 


\section{Introdução}

A Organização Mundial da Saúde (OMS) estima que, em 2008, cerca de 17,3 milhões de pessoas morreram devido a doenças cardiovasculares, dentre as quais 7,3 milhões em decorrência de doenças coronarianas ${ }^{1}$. A OMS destaca que as doenças cardiovasculares representam quase um terço dos óbitos em todo o mundo, dos quais $80 \%$ ocorreram nos países em desenvolvimento e acometeram igualmente homens e mulheres. Nesses países as pessoas estão mais expostas a fatores de risco e possuem menos acesso a serviços de saúde efetivos e equitativos, que incluem a detecção precoce de doenças cardiovasculares. No Brasil, a mortalidade por essas enfermidades representou 32,4\% dos óbitos no período de 1990 a 2009 , com taxas de mortalidade específica em torno de 46,8 por 100 mil habitantes. De 1993 a 1997, as internações por essas doenças corresponderam a $1,0 \%$ do total e a $3,3 \%$ dos gastos do SUS, sendo a angina responsável por $53,3 \%$ e o infarto por $26,6 \%$ das causadas por doenças isquêmicas do coração; as internações por infarto foram mais comuns em homens e por angina em mulheres ${ }^{2}$. A prevalência da hipertensão, sobrepeso e fumo, fatores de risco para doenças cardiovasculares, é de $24,4 \%, 46,6 \%$ e $15,5 \%$ respectivamente 3 .

$\mathrm{O}$ acesso ao diagnóstico precoce, à prevenção e ao tratamento adequado após a ocorrência de um infarto agudo do miocárdio ${ }^{4}$ tem contribuído para a redução da mortalidade por doenças cardiovasculares, especialmente nos países desenvolvidos.

Nos EUA são realizados anualmente mais de 1 milhão de procedimentos de cirurgia cardíaca, incluídos angioplastias e cirurgias de revascularização ${ }^{5}$. Estima-se que através desses procedimentos haveria um aumento de $7 \%$ na sobrevida dos pacientes ${ }^{6}$. As taxas de procedimentos de revascularização (angioplastia + cirurgia de revascularização) variaram, no período de 2004 a 2008, entre os países da OCDE, sendo maiores nos EUA, Bélgica, Hungria e Alemanha, e menores, em Portugal, Irlanda, Reino Unido e Suíça. Nos EUA, em 2008, as taxas de angioplastia e cirurgia de revascularização foram respectivamente, 168 e 367 por 100 mil habitantes e no Canadá, nesse mesmo ano, foram 108 e 70 por 100 mil habitantes $^{7}$. O uso da angioplastia tem aumentado rapidamente desde 1990 na maioria dos países da OCDE, correspondendo a 75\% do total de procedimentos de revascularização ${ }^{8}$. Nos EUA, a taxa anual de realização de cirurgias de revascularização tem diminuído em comparação com a angioplastia, e essa diminuição estaria relacionada a múltiplos aspectos, tais como a introdução de tecnologias competitivas, avanços nas técnicas cirúrgicas de revascularização, publicação de achados de ensaios clínicos ou de diretrizes clínicas. Dentre as possíveis causas para as variações entre os países da OCDE estão as diferenças na incidência e prevalência de doenças isquêmicas do coração, na capacidade de oferecer e pagar por esses procedimentos, nas diretrizes clínicas e na codificação e registro dessas práticas. Estudos de utilização dos serviços de saúde mostraram que as taxas de cirurgia de revascularização eram menores nos grupos em situação sociogeográfica mais desvantajosa ${ }^{9-12}$. Achados de estudos norte-americanos indicam que o acesso de mulheres e de pessoas da raça negra às cirurgias de revascularização e angioplastia é menor, quando comparado aos de homens brancos, sendo que no período de 1993 a 2001 houve um aumento na diferença absoluta na taxa de realização de angioplastia entre os homens brancos e os demais grupos ${ }^{13}$.

No Brasil, dentre as diversas cirurgias cardíacas realizadas pelo Sistema Único de Saúde (SUS), a mais frequente é a cirurgia de revascularização miocárdica, realizada por mais de uma centena de equipes, tanto em hospitais públicos como em filantrópicos ou privados. Entre 2005 e 2007 uma grande disparidade regional foi registrada na relação do número de cirurgias realizadas por número de habitantes. As regiões Sul e Sudeste, mais desenvolvidas em termos socioeconômicos e com o maior número de leitos e hospitais especializados disponíveis, concentravam o maior número de operações por habitantes quando comparadas com o Norte e o Nordeste, correspondendo a $77 \%$ do total de cirurgias realizadas ${ }^{14}$.

As taxas internação por angioplastia e cirurgia de revascularização vêm sendo usadas como proxies do acesso a serviços de alta complexida$\mathrm{de}^{15-17}$. Os dados referentes ao período 2002 a 2010 indicam que houve um acréscimo substancial no uso desses procedimentos com diferenças importantes entre as grandes regiões e as UF. O objetivo deste trabalho é analisar em maior profundidade a evolução dessas taxas e discutir quais seriam as possíveis causas associadas às desigualdades regionais como, por exemplo, razão de sexo, percentual de população idosa, distribuição espacial da oferta de serviços especializados, e cobertura da população por plano de saúde privado. 
A avaliação do acesso aos serviços de saúde distingue, como apontam Travassos e Martins ${ }^{18}$, com base no modelo de Andersen, os conceitos de acesso potencial e acesso realizado, no qual o acesso potencial caracteriza-se pela presença no âmbito dos indivíduos de fatores capacitantes do uso de serviços, enquanto acesso realizado representa a utilização de fato desses serviços e é influenciado por fatores outros além dos que explicam o acesso potencial. O conceito de acesso potencial incorpora, como apontam as autoras, fatores individuais que representam apenas um subconjunto dos fatores que explicam o acesso realizado (uso), pois estes incluem também os fatores predisponentes, as necessidades de saúde, além de fatores contextuais. Neste estudo, as taxas de utilização de cirurgia cardiovascular foram empregadas como proxy para estimar o acesso aos serviços de média e alta complexidade de saúde ${ }^{19}$.

Foram calculadas as taxas padronizadas de realização de angioplastia e cirurgia de revascularização por sexo e idade por 100 mil habitantes de 20 anos e mais, no período 2002 a 2010.

O numerador das taxas de angioplastia foi obtido através dos dados do SIH/SUS considerando os seguintes procedimentos: angioplastia coronariana; coronarioplastia com implante duplo de prótese de sustentação intraluminar; angioplastia em enxertos coronarianos; angioplastia em enxertos coronarianos com implante de próteses e angioplastia coronariana primária incluso cateterismo. No caso da cirurgia de revascularização foram considerados os procedimentos: revascularização miocárdica com uso de extracorpórea; revascularização miocárdica sem uso de extracorpórea; revascularização miocárdica com uso de extracorpórea com dois ou mais enxertos inclusive arterial; revascularização miocárdica sem uso de extracorpórea com dois ou mais enxertos inclusive arterial; troca valvar com revascularização miocárdica. Os procedimentos selecionados tanto para angioplastia quanto para cirurgias de revascularização foram os mesmos utilizados por outros autores que trabalharam esse tema ${ }^{14-16}$.

A população de 20 anos e mais das unidades federadas, regiões e Brasil, estimada pelo IBGE a partir das projeções do Censo Demográfico de 2000 para o período 2002 - 2006 foi utilizada como denominador para o cálculo das taxas. A partir de 2007 as estimativas populacionais estão baseadas na Contagem de 2007 e no censo de 2010.
Para analisar as desigualdades geográficas foram usados alguns indicadores demográficos, oferta de serviços disponíveis ao SUS (AMS 2009), recursos humanos disponíveis ao SUS (CNES) e a proporção de população com cobertura por plano privado de saúde (ANS - 2010). O indicador demográfico selecionado foi a razão entre a proporção de pessoas com 60 anos e mais e a população de 20 a 59 anos. Os indicadores de oferta incluem os leitos cirúrgicos e os leitos de UTI e UCO disponíveis ao SUS por 100 mil habitantes, calculados a partir dos 495 estabelecimentos (serviços próprios ou terceirizados que realizam cirurgia cardíaca) cadastrados na AMS de 2009. O indicador de recursos humanos empregado foi o número de cirurgiões cardiovasculares registrados no CNES em 2010. A proporção de pessoas com plano de saúde privado foi calculada considerando-se apenas a população de 20 anos e mais segundo a ANS em 2010.

\section{Resultados}

No Brasil, as taxas padronizadas de internação por angioplastia na população de 20 anos e mais apresentaram uma tendência de crescimento, passando de 27,5 por 100 mil habitantes em 2002 para 39 por 100 mil em 2010. As taxas mais altas foram observadas na região Sul, que em alguns anos do período analisado foram superiores a 70 por 100 mil habitantes. Na região Sudeste, as taxas foram semelhantes à média nacional. Nas outras regiões verificam-se taxas inferiores à média nacional, sendo que na região CentroOeste verificou-se um aumento importante a partir de 2008 (Tabela 1).

Deve ser ressaltado que no interior das grandes regiões há diferenças importantes entre as taxas estaduais. Considerando apenas o ano de 2010, há taxas acima da média nacional em estados de todas as regiões, sendo que, no caso da região Sul os três estados estão entre os que apresentam os maiores valores, destacando-se o Rio Grande do Sul (85,7 por 100 mil habitantes). No Sudeste, o Rio de Janeiro foi único estado com valores inferiores à média nacional. Outros estados com taxas superiores à média nacional foram Amapá, Rio Grande do Norte e Cearán ${ }^{17}$.

As taxas padronizadas de revascularização na população de 20 anos e mais diferem das taxas padronizadas de angioplastia ao apresentarem uma leve tendência de decréscimo, passando de cerca de 20, em 2002, para 15 por 100 mil habitantes em 2010. Entretanto, de modo similar 
ao observado nas angioplastias, a região Sul apresentou as taxas mais altas de revascularização. Nas regiões Sudeste e Centro-Oeste, as taxas foram próximas à média nacional, sendo que a tendência de diminuição foi maior no Centro-Oeste. As outras regiões apresentaram taxas inferiores à média nacional, verificando-se um quadro de estabilidade nas mesmas ao longo do período (Tabela 2).

No nível estadual, a evolução do indicador de revascularização foi mais estável que a observada em relação às taxas de angioplastia. Considerando o ano de 2010, a maior parte dos estados das regiões Norte e Nordeste apresentou taxas inferiores à média nacional, sendo que apenas no caso do Piauí e Rio Grande do Norte o indicador foi um pouco superior. No Sudeste, os maiores valores (acima da média do Brasil) foram observados nos estados do Espírito Santo (17,2 por 100 mil) e São Paulo (21,8 por 100 mil). Na região Sul os três estados apresentam valores próximos ou superiores aos verificados na média nacional. Cabe destacar que no estado de Paraná foi observado o valor mais alto entre todas as UF (34,4 por 100 mil habitantes). Na região Centro-Oeste destaca-se Mato Grosso do Sul (22,7 por 100 mil) e o Distrito federal com um valor pouco superior à media nacional ${ }^{16}$.
Quando se analisa a proporção de angioplastia entre procedimentos de cirurgia cardíaca no período de 2002 a 2010, nota-se um aumento significativo nessa proporção em todas as regiões, que tende a convergir para algo em torno de 70\% em 2010. Em 2010, os únicos estados com valores inferiores a 50\% foram Roraima e Piauí (entre 18 e 20\%) (Gráfico 1).

Considerando-se o total de procedimentos para cirurgia cardiovascular (angioplastia + revascularização), em 2010, as taxas padronizadas por idade e sexo (Tabela 3 ) entre pessoas com 20 anos e mais variaram de 21,8 - na região Norte a 94,2 por 100 mil habitantes na região Sul, sendo que para o Brasil essa taxa estava em torno de 54 internações por 100 mil habitantes, valor muito semelhante ao da região Sudeste. As regiões Nordeste e Centro Oeste apresentaram taxas inferiores à taxa nacional. Em todas as regiões a razão entre as taxas por sexo é similar e tendem a ser duas vezes maiores entre os homens.

No que se refere à oferta de serviços, verifica-se que a região Sul, onde foram observadas as taxas mais altas de internação por cirurgia cardíaca, possui as maiores taxas de leitos cirúrgicos, leitos de UTI e UCO disponíveis ao SUS, assim como a maior taxa de cirurgiões cardiovasculares por cem mil habitantes que tem vínculo com o SUS.

Tabela 1. Taxas padronizadas para internações por angioplastia na população de 20 anos e mais. Brasil e grandes regiões, 2002 - 2010.

\begin{tabular}{lrrrrrrrrr}
\hline \multicolumn{1}{c}{ Região } & $\mathbf{2 0 0 2}$ & $\mathbf{2 0 0 3}$ & $\mathbf{2 0 0 4}$ & $\mathbf{2 0 0 5}$ & $\mathbf{2 0 0 6}$ & $\mathbf{2 0 0 7}$ & $\mathbf{2 0 0 8}$ & $\mathbf{2 0 0 9}$ & $\mathbf{2 0 1 0}$ \\
\hline Norte & 3,1 & 4,2 & 6,5 & 9,4 & 16,0 & 12,8 & 15,1 & 19,1 & 14,7 \\
Nordeste & 12,2 & 14,2 & 16,2 & 18,1 & 19,2 & 19,2 & 20,9 & 22,4 & 21,9 \\
Sudeste & 30,2 & 31,7 & 32,1 & 34,2 & 39,6 & 34,3 & 34,8 & 39,5 & 41,1 \\
Sul & 54,9 & 58,5 & 61,3 & 64,9 & 74,9 & 65,4 & 69,0 & 70,0 & 71,2 \\
Centro Oeste & 21,2 & 17,7 & 18,6 & 21,7 & 24,5 & 21,0 & 22,6 & 26,2 & 33,0 \\
Brasil & 27,5 & 29,1 & 30,4 & 32,8 & 37,6 & 33,6 & 35,1 & 38,2 & 39,0 \\
\hline
\end{tabular}

Tabela 2. Taxas padronizadas para internações por cirurgia de revascularização na população de 20 anos e mais. Brasil e grandes regiões, $2002-2010$.

\begin{tabular}{lrrrrrrrrr}
\hline \multicolumn{1}{c}{ Região } & $\mathbf{2 0 0 2}$ & $\mathbf{2 0 0 3}$ & $\mathbf{2 0 0 4}$ & $\mathbf{2 0 0 5}$ & $\mathbf{2 0 0 6}$ & $\mathbf{2 0 0 7}$ & $\mathbf{2 0 0 8}$ & $\mathbf{2 0 0 9}$ & $\mathbf{2 0 1 0}$ \\
\hline Norte & 7,4 & 8,3 & 8,7 & 8,4 & 9,6 & 7,9 & 8,3 & 8,9 & 7,1 \\
Nordeste & 9,5 & 11,2 & 10,4 & 10,3 & 11,5 & 10,7 & 9,5 & 9,9 & 9,1 \\
Sudeste & 20,1 & 20,0 & 19,5 & 20,5 & 22,1 & 18,2 & 17,7 & 18,3 & 16,8 \\
Sul & 27,9 & 31,1 & 31,5 & 30,3 & 29,7 & 25,5 & 22,8 & 24,2 & 22,7 \\
Centro Oeste & 20,4 & 19,9 & 17,5 & 17,5 & 17,6 & 14,0 & 12,5 & 12,9 & 12,0 \\
Brasil & 17,9 & 18,9 & 18,3 & 18,6 & 19,6 & 16,7 & 15,6 & 16,3 & 14,9 \\
& & & & & & & & &
\end{tabular}


Tabela 3. Distribuição das internações por cirurgia cardíaca (angioplastia + cirurgias de revascularização), oferta de leitos, cirurgiões cardíacos e cobertura por plano de saúde segundo Ufs e Grandes Regiões, Brasil, 2010.

\begin{tabular}{|c|c|c|c|c|c|c|c|c|c|}
\hline UF/GR & $\begin{array}{c}\text { No de } \\
\text { internações }\end{array}$ & $\begin{array}{c}\text { Taxas } \\
\text { padronizadas } \\
\text { de internações } \\
(100 \text { mil hab. })\end{array}$ & $\begin{array}{c}\text { Razão } \\
\text { taxas } \\
\text { Masc/ } \\
\text { Fem }\end{array}$ & $\begin{array}{c}\% 60 \\
\mathrm{e} \\
\text { mais }\end{array}$ & $\begin{array}{c}\text { Leitos_SUS } \\
(100 \mathrm{mil} \\
\text { hab.) }\end{array}$ & $\begin{array}{l}\text { Leitos } \\
\text { UTI/UCO } \\
(100 \mathrm{mil} \\
\text { hab. })\end{array}$ & $\begin{array}{l}\text { Leitos } \\
\text { cirúrgicos } \\
(100 \mathrm{mil} \\
\text { hab. })\end{array}$ & $\begin{array}{l}\text { Cirurgiões } \\
\text { CV por } \\
100 \text { mil } \\
\text { hab. }\end{array}$ & $\begin{array}{l}\text { Cobertura } \\
\text { plano de } \\
\text { saúde } \\
\text { privado }\end{array}$ \\
\hline Norte & 1828 & 21,8 & 2,2 & 6,8 & 19,3 & 1,4 & 5,4 & 1,8 & 11,7 \\
\hline Nordeste & 11220 & 30,9 & 1,8 & 10,3 & 21,0 & 1,8 & 6,8 & 3,0 & 12,5 \\
\hline Sudeste & 38051 & 58,0 & 2,1 & 11,9 & 37,1 & 3,4 & 12,9 & 4,9 & 38,7 \\
\hline Sul & 21594 & 94,2 & 2,1 & 12,0 & 50,9 & 3,9 & 14,7 & 5,5 & 24,7 \\
\hline Centro-Oeste & 4245 & 45,2 & 2,1 & 8,8 & 29,4 & 2,6 & 8,9 & 4,0 & 17,8 \\
\hline Brasil & 76938 & 53,9 & 2,0 & 10,8 & 33,1 & 2,8 & 10,7 & 4,2 & 26,2 \\
\hline
\end{tabular}

Outro fator importante para entender as diferenças entre as taxas de internação para cirurgia cardíaca é a cobertura populacional por plano de saúde privado. Dados da ANS indicam que a proporção de população coberta por plano de saúde concentra-se nas regiões Sudeste e Sul. Entretanto, a relação entre as taxas de internação e a cobertura por plano de saúde privado nessas duas regiões é inversa, sendo que a região Sul, que tem maiores taxas de internação, apresenta menor proporção de população coberta por plano de saúde privado. Ao considerarmos somente a população não coberta por planos de saúde privados, a diferença entre as taxas de internação cai de $68 \%$ para $38 \%$, ainda favorável à região Sul.

\section{Discussão}

A comparação com os dados internacionais mostra que o Brasil tem taxas menores que as observadas nos países da $\mathrm{OECD}^{8}$, à exceção do Chile e do México. Porém, essas comparações devem levar em conta que os dados da OECD apresentam variações devidas ao sistema de classificação e registro das práticas, tais como a contagem do procedimento principal como procedimento único ou duplo (p.ex. angioplastia com stent) e a exclusão das cirurgias realizadas em hospitais privados ou hospitais-dia. No caso do Brasil, as taxas não contemplam as informações do setor privado não conveniado ao SUS. Além disso, devem 
ser levadas em conta, em comparações internacionais, as diferenças na estrutura da população.

Outras limitações dos dados brasileiros referem-se à questão do teto financeiro para pagamento de procedimentos de internação, o que pode influenciar o acesso às cirurgias de revascularização na rede pública ou conveniada ao SUS. Essa limitação tem sido minimizada em alguns municípios, que assumem os custos dos procedimentos que extrapolam o teto financeiro contratando os serviços de unidades de saúde privadas. Entretanto, esses dados não são registrados nos sistemas de informação do Ministério da Saúde, o que acarreta uma subestimação das taxas de cirurgias realizadas nessas localidades quando se utiliza os sistemas de informações nacionais. Além disso, as taxas de cirurgias cardíacas apresentam oscilações ao longo do tempo em decorrência das variações nas estimativas populacionais. A queda observada nas taxas de internação de angioplastia e revascularização em todas as regiões no ano de 2007 deve-se a mudanças na estimativa populacional, já que a Contagem Populacional pelo IBGE em 2007 acrescentou 10 milhões de pessoas de 20 anos e mais em relação ao número estimado a partir do censo de 2000 para o ano de 2006.

Apesar dessas limitações, os achados do estudo mostram-se consistentes em alguns aspectos quando comparados aos dados de outros países. A razão entre os sexos na realização das cirurgias é compatível com os achados da literatura que mostram maiores chances de homens serem submetidos a cirurgias de revascularização. Do mesmo modo, o efeito de substituição, com o aumento da proporção de angioplastias no total das cirurgias cardíacas, também é observado em outros países e pode estar relacionado a vários fatores, dentre eles o aumento do número de especialistas que realizam o procedimento cirúrgico ou de exames diagnósticos (cateterismo cardíaco) realizados.

$\mathrm{Na}$ comparação das taxas padronizadas por idade e sexo entre as grandes regiões do Brasil, além das diferenças marcantes no eixo Norte Sul, o que chama atenção é a manutenção de um padrão estável dessas taxas ao longo do período analisado. Essa estabilidade aponta para uma estratégia nacional de financiamento que não leva em conta as necessidades regionais em termos de oferta de serviços de saúde de alta complexidade.
A despeito da proposta de uma Política Nacional de Atenção Cardiovascular de Alta Complexidade, definida pela portaria GM/MS no 1.169 / $2004^{20}$, e dos recursos disponibilizados pelo Ministério da Saúde para a implantação de uma rede de assistência cardiovascular, a detecção precoce de doenças cardiovasculares e a ampliação do acesso à atenção de média e alta complexidade ainda não são suficientes para atender as necessidades regionais de saúde da população no que se refere à cirurgia cardíaca.

A proposta do Ministério da Saúde do Pacto pela Saúde, retificada posteriormente pelo Decreto $7508^{21}$, concebe uma proposta de regionalização apoiada em dois pressupostos básicos - a garantia do acesso aos serviços de saúde, buscando atender às situações diversas com uma melhor e mais rápida resposta aos desejos e necessidades dos usuários. Além disso, preconiza a implantação racional dos recursos de saúde, que devem ser organizados segundo uma lógica hierárquica, com o objetivo de otimizar os gastos com base nos ganhos de eficiência decorrentes da articulação das vantagens da provisão local com economia de escala nas ações e serviços prestados ${ }^{22}$.

Achados de investigações prévias mostram a importância da análise dos dados segundo as regionais de saúde para a avaliação e a melhoria do acesso aos procedimentos de média e alta complexidade ${ }^{15,23}$. No caso das cirurgias cardíacas, a constituição de redes assistenciais hierarquizadas é uma estratégia importante para garantir a qualidade do cuidado e a otimização dos custos operacionais.

\section{Colaboradores}

F Viacava, S Porto, J Laguardia, RS Moreira e MAD Ugá participaram de todas as etapas de elaboração deste texto. 


\section{Referências}

1. World Health Organization (WHO). [site na Internet]. [acessado 2012 out 20]. Disponível em: http:/ http://www.who.int/mediacentre/factsheets/fs310/en/

2. Brasil. Ministério da Saúde (MS). Inquérito Domiciliar sobre Comportamentos de Risco e Morbidade Referida por Doenças e Agravos não Transmissíveis: Brasil, 15 capitais e Distrito Federal, 2002/2003. Rio de Janeiro: INCA; 2004.

3. DATASUS. Dados de mortalidade, hipertensão, sobrepeso. [site na Internet]. [acessado 2012 out 20]. Disponível em: http://tabnet.datasus.gov.br/cgi/dh.exe? vigitel/vigitel09.def

4. Idänpään-Heikkilä UM, Lambie L, Mattke S, MClaughlin V, Palmers H, Tu JV. Selecting indicators for the quality of cardiac care at the health system level. Int J Qual Health Care 2006; 18(Supl. 1):39-44

5. Epstein AJ, Polsky D, Yang F, Yang L, Groeneveld PW. Coronary Revascularization Trendsin the United States, 2001-2008. JAMA 2011; 305(17):1769-1776

6. Gibbons RJ, Fihn SD. Coronary Revascularization: New Evidence, New Challenges. Ann Intern Med 2007; 147(10):732-734.

7. Canadian Institute for Health Information (CIHI). Health Indicators 2010. Toronto: CIHI; 2011.

8. Organization for Economic Co-operation and Development (OECD). Health at a Glance 2011: Health at a Glance 2011. Paris: OECD; 2011.

9. Ben-Shlomo Y, Chaturvedi N. Assessing equity in access to health care provision in the UK: does where you live affect your chances of getting a coronary artery bypass graft? J Epidemiol Community Health 1995; 49(2):200-204.

10. Ancona C, Agabiti N, Forastiere F, Arcà M, Fusco D, Ferro S, Perucci CA. Coronary artery bypass graft surgery: socioeconomic inequalities in access and in 30 day mortality. A population-based study in Rome, Italy. J Epidemiol Community Health 2000; 54(12):930-935.

11. Majeed A, Eliahoo J, Bardsley M, Morgan D, Bindman AB. Variation in coronary artery bypass grafting, angioplasty, cataract surgery, and hip replacement rates among primary care groups in London: association with population and practice characteristics. J Public Health Med 2002; 24(1):21-26.

12. Vanasse A, Niyonsenga T, Courteau J, Hemiari A. Access to myocardial revascularization procedures: Closing the gap with time? BMC Public Health 2006; 6:60.

13. Lucas FL, DeLorenzo MA, Siewers AE, Wennberg DE. Temporal Trends in the Utilization of Diagnostic Testing and Treatments for Cardiovascular Disease in the United States, 1993 - 2001. Circulation 2006; 113(3):374-379.
14. Piegas LS, Bittar OJNV, Haddad N. Cirurgia de revascularização miocárdica: resultados do Sistema Único de Saúde. Arq. Bras. Cardiol. 2009; 93(5):555560.

15. Pinto AMS, Pinheiro RS. Utilização de cirurgias cardíacas de alta complexidade no Estado do Rio de Janeiro numa perspectiva regionalizada: SIHSUS - 1999 a 2007. Cad Saude Coletiva 2010; 18(3): 445-455.

16. Piegas LS, Haddad N. Intervenção Coronariana Percutânea no Brasil. Resultados do Sistema Único de Saúde. Arq Bras Cardiol 2011; 96(4):317-324.

17. Brasil. Ministério da Saúde (MS). Pro-Adess - Avaliação do Desempenho do Sistema de Saúde. [site na Internet]. [acessado 2012 out 3]. Disponível em: http://www.proadess.cict.fiocruz.br/

18. Travassos C, Martins M. Uma revisão sobre os conceitos de acesso e utilização de serviços de saúde. Cad Saude Publica 2004; 20(Supl. 2):S190-S198.

19. Smith PC, Mossialos E, Papanicolas I, Leatherman S. Performance Measurement for Health System Improvement: Experiences, Challenges and Prospects. Cambridge: Cambridge University Press; 2010.

20. Brasil. Ministério da Saúde (MS). Portaria no 1.169/ GM em 15 de junho de 2004. Institui a Política Nacional de Atenção Cardiovascular de Alta Complexidade, e dá outras providências. Diário Oficial da União 2004; 16 jun.

21. Brasil. Presidência da República. Decreto no. 7.508 de 28 de junho de 2011. Regulamenta a Lei no 8.080, de 19 de setembro de 1990, para dispor sobre a organização do Sistema Único de Saúde - SUS, o planejamento da saúde, a assistência à saúde e a articulação interfederativa, e dá outras providências. Diário Oficial da União 2011; 29 jun.

22. Gadelha CAG, Machado, CV, Lima LD, BaptistaTWF. Saúde e territorialização na perspectiva do desenvolvimento. Cien Saude Colet 2011; 16(6):30033016.

23. Oliveira EXG, Carvalho MS, Travassos C. Territórios do sistema Único de Saúde: mapeamento das redes de atenção hospitalar. Cad Saude Publica 2004; 20(2):386-402.

Artigo apresentado em 30/04/2012

Aprovado em 17/07/1012

Versão final apresentada em 30/08/2012 